\title{
Penetrating Chest Wall Injury - A District Hospital Experience
}

\author{
Amirul Adib Ahmad Zakhi \\ Hospital Sungai Bakap, Pulau Pinang, 14200, Malaysia
}

\begin{abstract}
\section{Introduction}

Chest trauma constitutes $10 \%$ of trauma admission and $25 \%$ of trauma death globally. The management can be challenging even in a well-established trauma center. This is a report of our experience with penetrating chest wall injury in a district hospital setting.
\end{abstract}

\section{Clinical Summary}

A 16-year-old boy, motorcycle rider, involved in a motor-vehicle accident with a lorry.

Post trauma, he experienced breathlessness and sustained wound over zone 1 of the neck. Clinical assessment concluded the diagnosis of penetrating chest trauma complicated with right pneumothorax. Right chest tube, 3 sided occlusive dressing, cervical collar, oxygen support, IV antibiotic and analgesia were administered accordingly. The boy was subsequently transferred out to tertiary center for CT imaging and definitive management through multidisciplinary approach. He was then discharged from ICU to the general ward, after which he had an uneventful recovery and was discharged home in good condition.

Chest trauma can result in penetrating or blunt trauma. While blunt trauma is more common, penetrating trauma can be acutely life-threatening. This patient developed pneumothorax, one of the commonest complications, apart from hemothorax and hemopneumothorax. The site of chest wall injury, as in zone 1 of the neck in this patient, determines the subsequent management and the importance of identifying the hard and soft neck signs.

\section{Conclusion}

Appropriate and timely diagnosis of chest trauma is of great importance and correct diagnos is of the chest injury can decrease the mortality and morbidity. The key point in diagnosis of chest trauma is having a high index of suspicion of the probability of chest trauma presence in an injured patient.

\section{Keywords}

Emergency medicine, chest wall injury, pneumothorax 